PROPOSAL TO STUDY $\bar{p}$ INDUCED REACTIONS BY MEANS

OF THE $15^{\prime}$ BUBBLE CHAMBER, i.e.:

- $\overline{\mathrm{p}} \mathrm{d}$ interactions at $\sim 50$ and $\sim 80 \mathrm{GeV} / \mathrm{c}$

- $\bar{p}$ p interactions at $\sim 80 \mathrm{GeV} / \mathrm{c}$

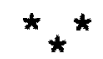

H. BRAUN, D. BRICK, F. ETIENNE, A. FRIDMAN* , J-P. GERBER, E. JEGHAM, P. JUILLOT, G. MAURER, A. MICHALON, M-E. MICHALON-MENTZER, C. VOLTOLINI

The Hydrogen Bubble Chamber Group of Strasbourg 
1. INTRODUCTION

As a continuation of the work done at Strasbourg we are proposing to study the $\bar{p}$ interactions at $\sim 50$ and $\sim 80 \mathrm{GeV} / \mathrm{c}$ and also $\bar{p}$ interactions at $\sim 80 \mathrm{GeV} / \mathrm{c}$. The exact values of the momenta are not very crucial although we would like to have the same value $(\sim 80 \mathrm{GeV} / \mathrm{c})$ for the $\bar{p} \mathrm{~d}$ and $\bar{p} \mathrm{p}$ experiments. Because of the lack of data in this momentum range each of the proposed runs will be interesting by itself as will be seen from the physics discussed below. But, we also have in mind the use of the results of the proposed experiment in order to make a more general study of $\overline{\mathrm{pN}}$ interactions, i.e.:

a). We want to study the incident momentum dependence of the $\bar{p} d$ interactions. The proposed experiment will then complete our various $\bar{p}$ studies carried out at the following momenta : 4-4.5-5-5.5-9.3-14.6 GeV/c. As discussed below the treatment of the data at all these momenta by a same method will avoid systematic errors complications.

b). We intend to make a comparison between $\bar{p} n$ and $\bar{p} p$ interactions to study primarly isospin effects. To achieve this we are interested in obtaining p p data at $\sim 80 \mathrm{GeV} / \mathrm{c}$ for which no data are yet available. The $\bar{p}$ and $\bar{p}$ data obtained with the same apparatus and analysed in the same conditions will allow us to carry out a meaningfull comparison of $\bar{p} p$ and $\bar{p}$ interactions properties.

As a first step we would be interested in a statistics corresponding to $50,000 \bar{p}$ interactions at both momenta and also to the same amount of $\bar{p} p$ interactions. The 15' bubble chamber is well adapted to the outlined physics program which we will discuss below. Indeed we will fully benefit from the advantages connected with the use of $15^{\prime}$ chamber which in the present experiment are:

- The possibility of measuring the momenta with a good accuracy

- The high probability of observing secondary interactions faciliting thus the identification of the outgoing particles

- The large size of the chamber which will increase the probability to observe the decay of strange particles

- The high $\gamma$-ray conversion rate $(\sim 20 \%)$ allowing us to obtain information on $\pi^{\circ}$ and also on $\Sigma^{\circ}$ production. 
In the following we will describe the most important physics aspects connected with the proposed experiment. First we will discuss the general features which can be studied with the pd part of the experiment (Section 2). Our main objective remains, however, to study these features as a function of the incident momentum, using also our previous $\bar{p} d$ data. In Section 3 we will examine the $\overline{\mathrm{p}} \mathrm{p}$ interactions and their comparison with $\overline{\mathrm{p}} \mathrm{n}$ interactions.

\section{THE $\overline{\mathrm{P}} \mathrm{d}$ INTERACTIONS AT $\sim 50$ AND $\sim 80 \mathrm{GeV} / \mathrm{C}$}

a). Topological cross sections and statistical moments

It is primarly in the determination of these quantities that the sys-. tematic errors wi.11 be of importance. Our recent results in the $5.55-14.6 \mathrm{GeV} / \mathrm{c} \mathrm{re-}$ gion $^{(1)}$ (see also Fig.1) has shown that systematic errors are rather difficult to estimate. In the framework of the impulse approximation, however, these errors are expected to be nearly independent on the incident momentum. Therefore it seems to us essential that statistical moments and multiplicity distributions should be determined by the same experimental methods. Using our previous data $(4.5-15 \mathrm{GeV} / \mathrm{c})$ we will be able to carry out a meaningfull study of the incident momentum dependence of the $\bar{p}$ multiplicity distributions.

Among other things we will also see :

- If the dispersion $D$ and the average $\langle n\rangle$ of the charged multiplicity distributions are related through a linear rule as for $\mathrm{pp}^{(2)}$ and $\bar{p}^{(1)}$ interactions

- If the data still obey the early KNo scaling as shown by the $\bar{p}$ interactions in the $5.55-14.6 \mathrm{GeV} / \mathrm{c}$ region (Fig.2)

- If, as for lower incident momenta, the average $\langle n\rangle$ for $\bar{p} n$ is smaller than for $\bar{p}$ in contrast to the dispersion $D$ which is greater for $\bar{p} n$ than for $\bar{p}$.

b). Correlation between the production of neutral and charged particles

Recently the production of the average number of neutral pions as a function of the charged multiplicity has been studied (3) gibing information about the multiparticle production mechanism ${ }^{(4)}$. Profiting from the relatively high $\gamma$-ray conversion rate $(\sim 20 \%)$ in the $15^{\prime}$ bubble chamber, we will study the above mentioned correlation. We plan also to make similar studies when the neutral par- 
ticle will be this time $n, \bar{n}, \Lambda, \bar{\Lambda}, k^{\circ}$ and $\vec{k}^{\circ}$. We will here benefit from the large size of the chamber. Indeed some outgoing $n, \bar{n}$ will be identified through their secondary interactions whereas the strange particles will be recoignzed whenever they decay in the bubble chamber.

c). Non annihilation channels

A search for baryonic and mesonic resonances in non annihilation channels will be carried out. In particular, we expect to study the four constraints events as the results obtained from the bare 30-inch bubble chamber have shown that fitting of these events is indeed possible ${ }^{(5)}$. Here the situation is more favorable as we expect to measure the momenta of the tracks with a better accuracy than in the 30-inch bare bubble chamber. The leading particle effect will also be studied and compared with pp data.

d). Multiparticle production phenomena

At high energy the multiparticle production contribute to an important part of the inelastic cross section. Except for the multiplicity distribution already discussed above we will also study these production phenomena using, among other things, multivariable techniques as proposed some years ago(6). In the same manner as made for lower $\bar{p}$ incident momenta ${ }^{(7)}$ we will search for multivariable distributions sensitive to the production mechanism. To do this we need to know at least the momenta of the charged tracks. The $15^{\prime}$ chamber will allow us to proceed with such an analysis using in particular the four constraint events.

e). Diffraction dissociation

By using the non annihilation reactions we will be able to investigate the diffraction dissociation of the neutron target. This will be made by measuring the low momentum tracks associated with the neutron vertex in which a proton can be recognized. If instead of a proton a neutron is emitted we will only take the events in which the $n$ produces a secondary interaction, allowing its identification. We also expect to obtain some information on the double diffraction dissociation processes. 
f). Single particle distributions and inclusive reactions

We will also study the various single particle distribution obtained in inclusive and semi-inclusive reactions. In particular we will study the reactions

$$
\begin{aligned}
\bar{p} n & +\bar{p} x \\
& +p x \\
& +\pi^{ \pm} x \\
& +\overline{\Delta x} \\
& \rightarrow \Delta x
\end{aligned}
$$

The importance of quantum number exchanged in the $t$-channel will be stressed by comparing these reactions with pp and $\bar{p}$ data. Furthermore the identification of some of the produced $n$ and $\bar{n}$ will allow us to study the $\bar{p} n+n X$ and $\bar{p} n+\bar{n} x$ reactions.

\section{g) Strange particle production}

One of the most striking results obtained from survey experiments with the $30^{\prime \prime}$ bubble chamber is the rapid raising of the strange particle production (see Fig.3). If one assumes that strange particle production in pp and pn interactions are nearly equal we will obtain a rather important sample of events having strange particles in the final state (see Table I). As already stated above the $15^{\prime}$ chamber is particularly suitable for studying strange particle production. Moreover the observation of $\Lambda$ and $\bar{\Lambda}$ decays will also allow us to obtain polarization information.

h). Annihilation channels

We intend to study the four constraint annihilation channels if such annihilation processes take place in the $50-80 \mathrm{GeV} / \mathrm{c}$ region. Information about of the total annihilation cross section will also beobtained, using the high probability to observe secondary interaction for estimating the $\bar{p} n \rightarrow p p x, \bar{p} n x, \bar{n} p X$, nnX cross section ( $X$ means anything). If one assumes that the annihilation cross section is falling with the c.m. energy $\sqrt{\mathrm{S}}$ according to $\mathrm{s}^{-0.6}$ as shown by low energy data ${ }^{(8)}$, one would obtain a cross section of 5.9 and $3.9 \mathrm{mb}$ at 50 and 80 
$\mathrm{GeV} / \mathrm{c}$, respectively. As shown by Table I this will correspond here to a considerable number of events enabling to study the annihilation processes. We also expect to obtain some information about annihilations reactions containing $\mathrm{K}^{ \pm}$, $\mathrm{K}^{\circ}$ and $\widehat{\mathrm{K}}^{\circ}$ in the final state.

i). Coherent reactions

By coherent reactions we mean here reactions in which a deuteron is present in the final state. The most simple reaction is of course the elastic $\overline{\mathrm{p} d} \rightarrow \overline{\mathrm{p}} \mathrm{d}$ reaction from which knowledge about $\overline{\mathrm{p}} \mathrm{n}+\overline{\mathrm{p} n}$ can be obtained $(10,11)$. Although elastic scattering is generally studied in counters experiments we will measure the $\bar{p} d \rightarrow \bar{p} d$ scattering as a by-product of this experiment. In addition, we will obtain information about the four constraint inelastic coherent reactions. This will allow us to study the isospin $I=1 / 2$ systems recoiling against the deuteron. The compatibility of the data with helicity conservation in the $s$ or $t$ channels will also be examined.

j). Miscellaneous

In addition to the physics program discussed above, let us enumerate some additional subjects which are of interest and will be studied in the proposed experiment :

- Study of two particle correlation feature

- Study of reactions having two identified $\pi^{\circ}$ in the final state

Although the statistics will be small we hope to measure at least the $2 \pi^{\circ}$ angular correlation.

\section{THE $\vec{P} P$ INTERACTIONS AT $\sim 80 \mathrm{GeV} / \mathrm{c}$}

As a first step we will make a general study of the $\bar{p}$ interactions at $\sim 80 \mathrm{GeV} / \mathrm{c}$. Except for coherent reactions we will investigate the same physical problems as discussed for the $\bar{p}$ interactions. Then we will carry out a systematic comparison between the $\overline{\mathrm{p}} \mathrm{p}$ and $\overline{\mathrm{p}} \mathrm{interactions}$ at the same incident momentum. The major points of interest can be summarized as follows.

a). Comparison of the charged multiplicity obtained from and $\bar{p}$ n interactions

We will examine the statistical moments and the multiplicity distributions obtained from $\overline{\mathrm{p} p}$ and $\overline{\mathrm{p} n}$ interactions in order to analyse their differences. 
At $14.6 \mathrm{GeV} / \mathrm{c}$ we noted for instance that the $\bar{p}$ and $\bar{p}$ topological cross sections tend to be distributed on the same smooth curve (1) (Fig. 4) when they are plotted as function of $\langle n\rangle$. We will see if this effect persists at high energy and eventually try to understand this phenomenon.

b). Correlation between the average number of produced neutral particles as function of $\langle n\rangle$

The pp data examined until now indicate that the average number of $\pi^{\circ}$ $\left[\left\{n_{0}\right\rangle\right]$ is related to the charged multiplicity $n$ through a linear rule: $\left\langle n_{0}\right\rangle=\alpha n+\beta^{(3)}$ (at least for moderate $n$ ). Here $\alpha$ and $\beta$ are parameters depending on the available c.m. energy. One possible explanation of this linear relation has been proposed considering the production as an interplay of two different mechanisms ${ }^{(11)}$. One of them leads to pion production concentrated around an average depending on the available c.m. energy while the other results from the constraints introduced by isospin conservation. In this approach differences between $\bar{p}$ p and $\bar{p}$ data should beobserved. In any case we will study quantitatively the influence of isospin effects on the correlation just mentioned. The same analysis will also be made with other neutral particles as $n, \vec{n}, \Lambda, \pi, k^{\circ}$ and $\overline{\mathrm{K}}^{\circ}$.

c). The annihilation channels

In $\bar{p}$ p annihilation at low energy a systematic cross section excess of the $\overline{\mathrm{p} p} \rightarrow \mathrm{m} \pi^{+} \mathrm{m} \pi^{-} \pi^{0}[\mathrm{~m}>2]$ over the $\overline{\mathrm{p}}_{\mathrm{p}}+\mathrm{m} \pi^{+} \mathrm{m} \pi^{-}$reactions has always been observed. It is not clear yet if this excess is due to a G-parity effect or to the fact that an extra $\pi^{\circ}$ is produced. The comparison between four constraint annihilation channels in $\overline{\mathrm{P}}(\mathrm{G}=+1)$ and $\overline{\mathrm{p}}(\mathrm{G}=-1)$ interactions will allow the clarification of this problem. The fact that both experiments will be made in similar conditions will facilitate the comparison procedure.

d): The impulse approximation model

We also intend to compare the four constraint events obtained from $\bar{p} p$ interactions with the same type of reactions extracted from the $\bar{p}$ interactions but having a spectator neutron in the final state. (Remember that a small momentum neutron can be identified through its secondary interactions). This will allow us to see to what extent the cross section calculations made for the pn reactions are reliable. Eventually we will calculate correction factors which 
may have an importance in the determination of the statistical moments derived from the $\bar{p}$ interactions.

e). The inclusive reactions

In order to stress the importance of the quantum numbers exchanged in the $t$-channel, we will compare the inclusive reactions :

$$
\begin{aligned}
& \bar{p} p+a+x \\
& \bar{p} n \rightarrow a+x^{\prime}
\end{aligned}
$$

Here a denoted the same outgoing particles while the symbols $X$ and $X^{\prime}$ mean anything.

f). The elastic $\bar{p}$ and $\bar{p}$ p cross sections

Although these reactions are usually studied with the counter technique, we will be able to study the $\overrightarrow{p N}$ elastic scattering as a by-product of the proposed experiment. By the same methods as those used at $5.55 \mathrm{GeV} / \mathrm{c}(9,10)$ we will compare the $\bar{p}$ and $\bar{p}$ differential cross section and determine eventually their cross-over point.

g). Isospin analysis

A cross channel isospin analysis can be performed on the $\bar{p} N \rightarrow N(\bar{N} \pi)$ and $\overline{\mathrm{pN}} \rightarrow \overline{\mathrm{N}}(\mathrm{N} \pi)$ channels by treating in the same way the $\overline{\mathrm{p}} \mathrm{p}$ and $\overline{\mathrm{p}} \mathrm{d}$ data at $\sim 50 \mathrm{GeV} / \mathrm{c}$.

\section{CONCLUSIONS}

In order to estimate the number of pictures and the number of events in each topology let us first consider the p p interactions at $80 \mathrm{GeV} / \mathrm{c}$. As stated above we would like to have $50,000 \bar{p}$ interactions. Using a fiducial region of $2.8 \mathrm{~m}$ and $3 \bar{p} /$ burst this will correspond to about 50,000 photographs. Assuming that the $\bar{p}$ and pp topological cross sections at $80 \mathrm{GeV} / \mathrm{c}$ are in the same ratio as the total $\bar{p}$ and $\mathrm{pp}$ cross sections we obtain the number of events belonging to each topology as given by Table I. One sees from this table that for $2 \leq \mathrm{n}<14$ we will get a significant sample in each topology. 
For estimating the $\bar{p}$ cross sections we assumed that the $\bar{p} p$ and $\bar{p} n$ topological cross sections are distributed on the same smooth curve as shown by the $\overline{\mathrm{pN}}$ data at $\sim 15 \mathrm{GeV} / \mathrm{c}(\mathrm{Fig} \cdot 4)$. Interpolating the estimated $\overline{\mathrm{p}} \mathrm{p}$ topological cross sections we then obtain the expected number of $\bar{p}$ events in each topology, for a total of $50,000 \bar{p}$ events. Also here this will correspond roughly to 50,000 pictures. Although only one third of the events have a visible spectator proton stopping in the chamber, we intend to use also the events with invisible spectator for studying the general features of the pn interactions.

The order that the experiment will be made is not essential although we would prefer to carry out the pp run first. This will allow us to set up the software needed for the $15^{\prime}$ with the pp experiment, the analysis of which is more simple than the $\bar{p} d$ one. The exact value of the beam momenta is not very crucial as we want to cover the lack of pn data in the $15-100 \mathrm{GeV} / \mathrm{c} \mathrm{region}$. What is however important for our proposal is that the highest momentum we are asking for should be the same for the $\overline{p d}$ and $\bar{p} p$ study.

The Strasbourg Group will, of course, give the full priority to this experiment if it is approved. This means that all our scanning and measuring capacity will be devoted to the analysis of these films. The Hydra system being already used in Strasbourg, the adaptation of the $15^{\prime}$ software could be rather fast. At the first stage we intend to measure the events with conventional measuring machines. The setting errors for these devices has been decreased by using a television camera coupled to the optics of the apparatus in order to magnify the film image and display it on a TV screen. The measurements of $200 \mathrm{GeV} / \mathrm{c}$ film has shown that we obtain very good results with our system. At the time that the experiment will be done 5 hand measuring machine will be equiped with this TV screen.

Sixteen scanning persons ( 6 full time and 10 half time) will work on the proposed experiment. Two programmers will work on the 15' foot software, one of them could spend some months at FNAL. In addition a few physicists intend to come for one or two months in order to learn how to operate the beam. 
R.e

1). Study of charged multiplicity distributions in $\bar{p}$ interactions at $5.55,9.3$ and $14.6 \mathrm{GeV} / \mathrm{c}$, A. Fridman et al., submitted to Physica1 Review

2). Wroblewski, Zakopane Lectures (1973)

3). F.T. Dao and J. Withmore, Phys. Letters, 46B, 252 (1973)

4). See for instance: E.L. Berger, D. Horn and C. Thomas, Phys. Rev. D7, 1412 (1973)

5). See for instance : H.H. Bingham et a1., Phys. Letters, 51B, 397 (1974) and M. Derrick et a1., Phys. Rev., D9, 1215 (1974)

6). M.C. Foster et al., Phys. Rev., D6, 3135 (1972)

M.C. Foster, R.J. Loveless ans S. Nussinov, Phys. Rev., D8, 3848 (1973)

7). G. Alexander, S. Dagan, A. Fridman, J. Grunhaus, S. Nussinov and Y. Oren, Phys. Rev., D9, 649 (1974)

8). J. Salava and U. Simak, Nucl. Phys., B69, 15 (1974)

9). H. Braun, A. Fridman, J-P. Gerber, J. Ma1ko, A. Michalon, C. Voltolini, Char1ton, W.A. Cooper and B. Musgrave, Nuc1. Phys., B54, 61 (1973)

10). H. Braun, D. Brick, A. Fridman, J-P. Gerber, E. Jegham, P. Juillot, C. Voltolini, Phys. Rev., D10, 9 (1974)

11). D. Horn and A. Schwimmer, Nucl. Phys., B52, 627 (1973) 
TABLE I - Distribution at $80 \mathrm{GeV} / \mathrm{c}$ of the number of $\bar{p}$ events as a function of the number of charged particle $(n)$ for a total number of 50,000 pp interactions. The number of annihilation events was estimated assuming that the total annihilation cross section behaves as $s^{-0.6}$ where $\sqrt{s}$ is the $\bar{p} p$ c.m. energy

\begin{tabular}{|c|c|}
\hline $\mathbf{n}$ & Number of events \\
\hline $0\left\{\begin{array}{c}\text { elastic } \\
\text { inelastic }\end{array}\right.$ & 440 \\
4 & 6400 \\
6 & 10900 \\
8 & 9950 \\
10 & 7230 \\
12 & 3900 \\
14 & 1420 \\
16 & 560 \\
annihilation & 200 \\
strange partic 1 and $\Lambda)$ & 5200 \\
(Kotal & 6400 \\
\hline \hline
\end{tabular}


TABLE II - Distribution at 50 and $80 \mathrm{GeV} / \mathrm{c}$ of the number of events as a function of the charged multiplicity $n$ for a total number of 50,000 pn events (including the events with invisible spectator proton). The results are obtained by estimating the topological pn cross section as described in the text. It was assumed that $\bar{p}$ p and $\bar{p}$ annihilation cross section are equal at a given incident momentum.

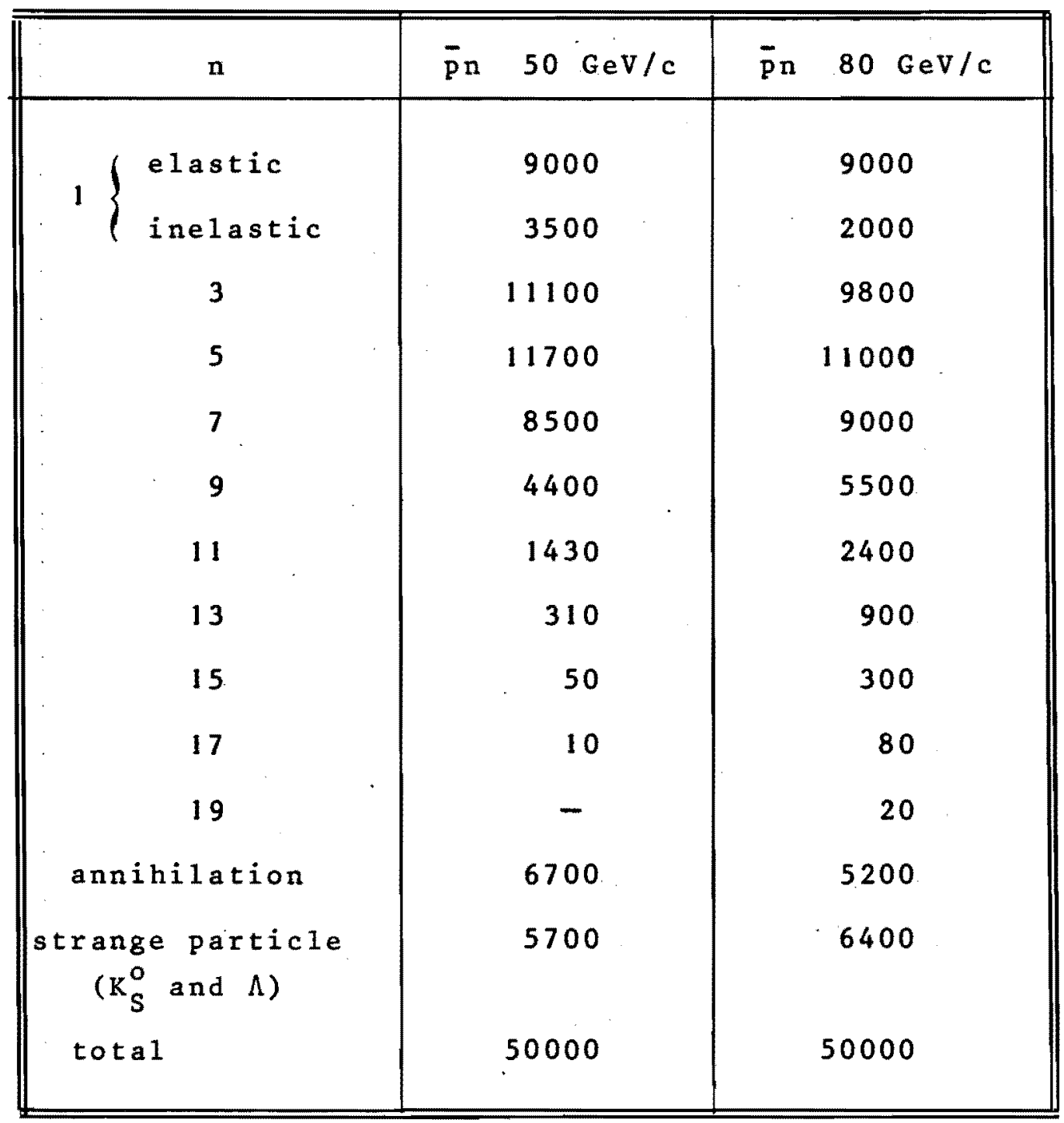




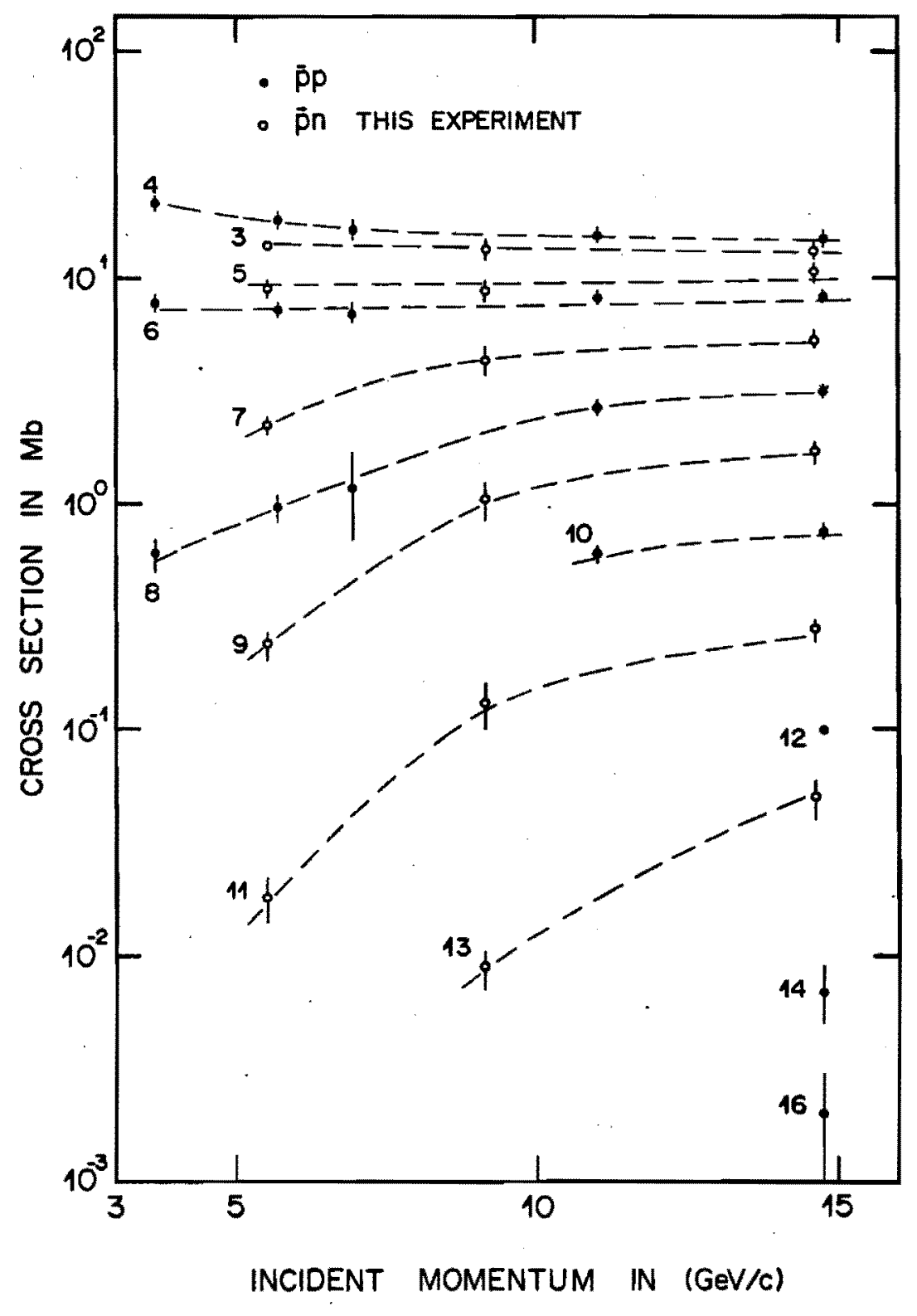

\section{Figure 1}

Distributions of the pon topological cross sections in the $3-15 \mathrm{GeV} / \mathrm{c}$ incident momentum region and comparison with $\bar{p}$ data [taken from reference (1)] 


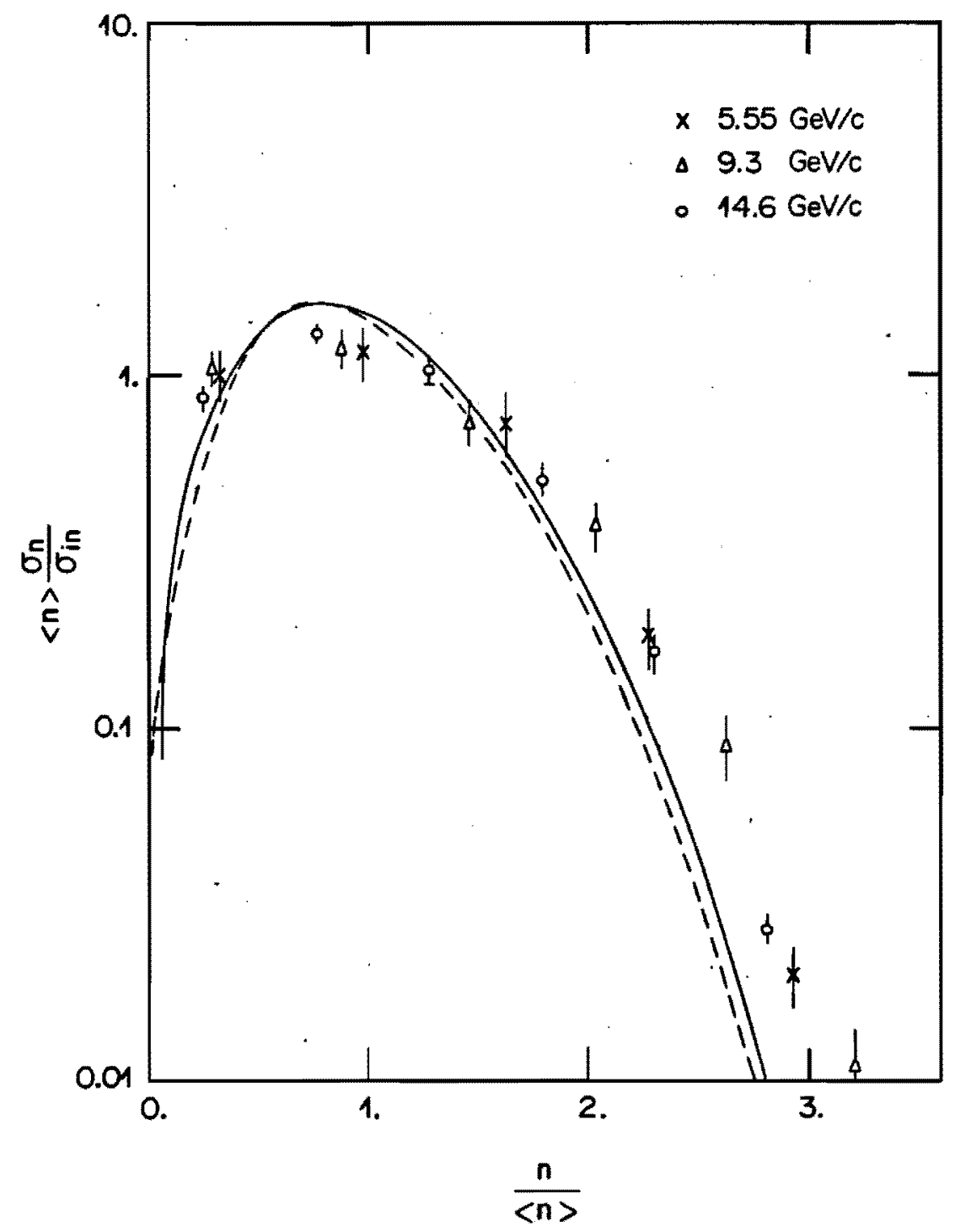

Figure 2

The distribution of $\langle n\rangle \sigma_{n} / \sigma_{\text {in }}$ versus $n /\langle n\rangle$ for the $\bar{p} n$ interactions at $5.55,9.3$ and $14.6 \mathrm{GeV} / \mathrm{c}$. The full and dashed lines are obtained by fitting the pp and $\bar{p} p$ data. Note that the scaling behavior seems to be different for $p p, \bar{p} p$ and $\bar{p} n$ interactions [reference (1)] 

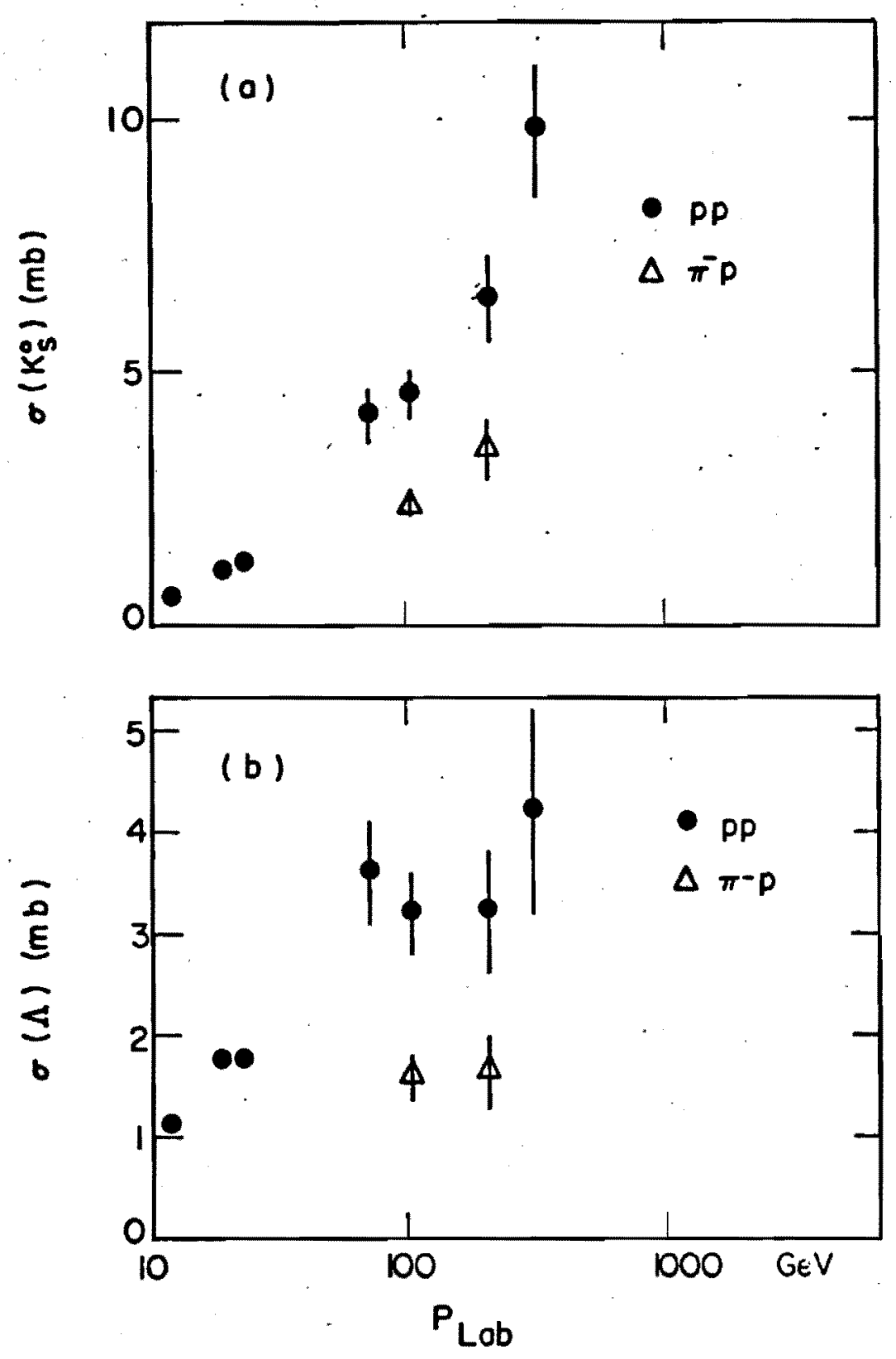

Figure 3

Cross section for $\mathrm{K}_{\mathrm{S}}^{\mathrm{O}}\left[\sigma\left(\mathrm{K}_{\mathrm{S}}^{\circ}\right)\right]$ and $\Lambda[\sigma(\Lambda)]$ production as function of $\mathrm{P}_{1 \mathrm{ab}}$, the incident momentum (taken from CERN SPSC/75-15 report) 


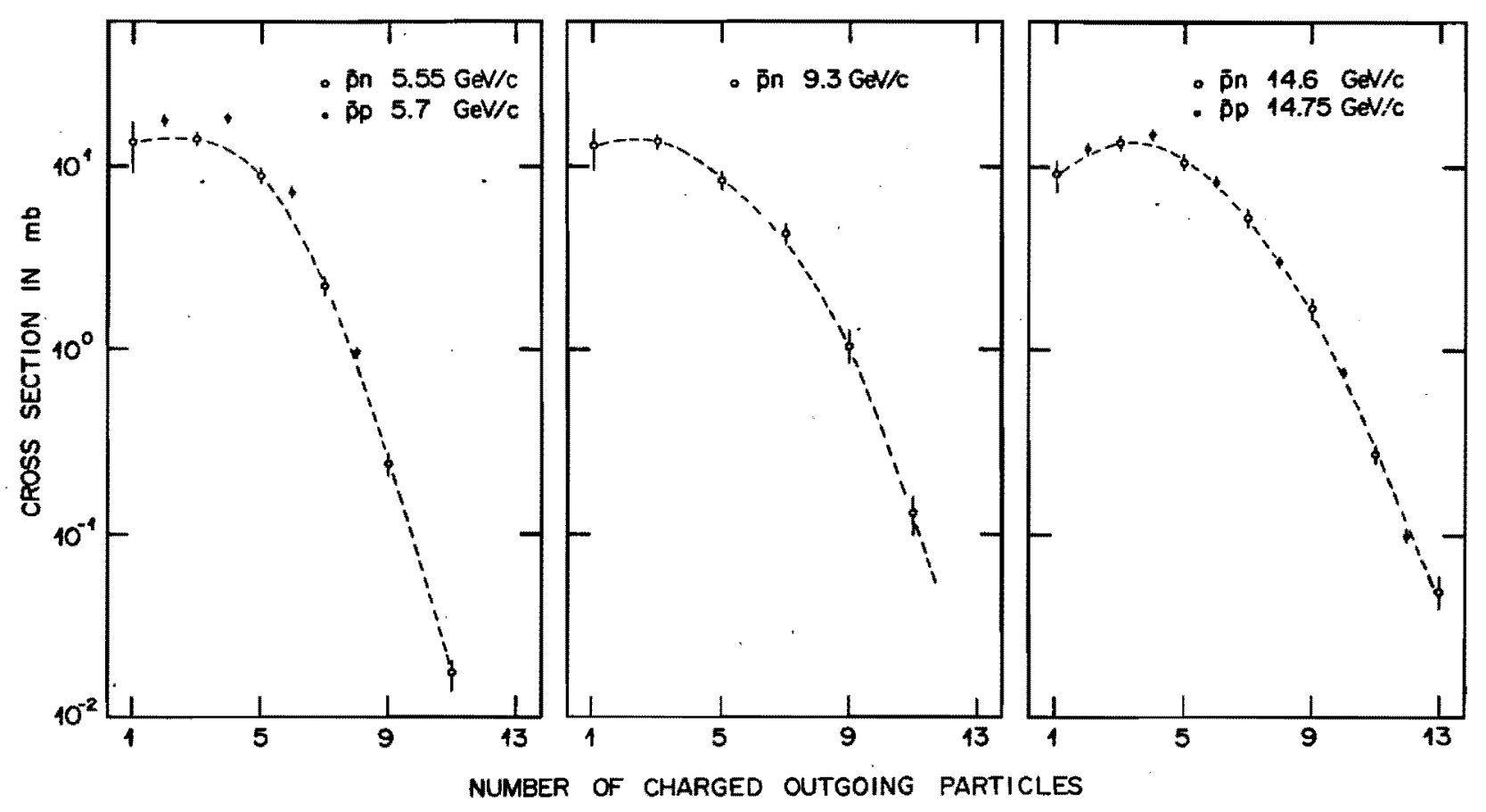

Figure 4

Comparison of the $\bar{p}$ topological cross sections plotted versus the number of charged outgoing particles with the available pp data. Note that at $\sim 15 \mathrm{GeV} / \mathrm{c}$ the $\overline{\mathrm{p}} \mathrm{p}$ and $\overline{\mathrm{p}}$ results tend to be distributed on a same smooth curve. The lines are drawn to guide the eye. 